\title{
QTL mapping of oil-related traits in sunflower from VNIIMK collection
}

\author{
Gubaev R. ${ }^{1 *}$, Boldyrev S. ${ }^{1}$, Chernova A. ${ }^{1}$, Martynova E. ${ }^{1}$, Kovalenko T. ${ }^{2}$, Goryunova S. ${ }^{1}$, \\ Goryunov D. ${ }^{1}$, Peretyagina T. ${ }^{2}$, Ben C. ${ }^{1}$, Gentzbittel L ${ }^{1}$., Demurin Y. ${ }^{2}$, Khaitovich P. ${ }^{1}$ \\ ${ }^{1}$ Skolkovo Institute of Science and Technology, Moscow, Russia \\ ${ }^{2}$ All-Russia Research Institute of Oil Crops, Krasnodar, Russia \\ *rim.gubaev@skoltech.ru
}

Key words: oil oxidative stability, genotyping-by-sequencing, genetic linkage maps, QTL mapping

Motivation and Aim: Tocopherols and oleic acid are components of sunflower oil that protect it against thermooxidation and significantly affect its quality. The tocopherol complex in plants includes four different forms, namely, $\alpha$-tocopherol, $\beta$-tocopherol, $\gamma$ tocopherol, and $\delta$-tocopherol. Oleic acid is a monounsaturated fatty acid that in combination with tocopherols significantly increases oil thermostability. Therefore, one of the pressing tasks in sunflower breeding is the creation of plants that would allow producing oil with balanced tocopherol composition and oleic acid content. To facilitate the identification of new loci connected with these traits, we performed QTL mapping based on the high-throughput sequencing data for sunflower plants.

Methods and Algorithms: Two F2 populations were obtained from two independent crosses of parents contrast in tocopherol composition and oleic acid content. Tocopherol composition was measured using thin-layer chromatography while the oleic acid content was measured by means of gas chromatography followed by mass spectrometry. The genotyping-by-sequencing was performed using the Illumina HiSeq platform. The Tassel-GBS pipeline was used to find genetic polymorphisms. For genetic map construction, the Kosambi mapping function was used to order the markers. For QTL mapping of loci associated with tocopherol composition and oleic acid content composite interval mapping approach was applied.

Results: Using the GBS approach and QTL mapping loci associated with $\alpha-, \gamma-$, and $\delta$ tocopherol were located on chromosome 8 while loci associated with $\beta$-tocopherol content were found on chromosomes 8 and 1. For oleic acid, a locus located on chromosome 14 was shown to be significantly associated.

Conclusion: The QTLs and corresponding SNPs identified will facilitate the markerassisted selection of sunflower as well as bring new knowledge on the genetic control of tocopherol composition and oleic acid content of the sunflower oil.

Acknowledgements: The reported study was funded by RFBR projects number 20-31690051. 